EPJ Web of Conferences 110,01067 (2016)

DOI: $10.1051 /$ epjconf/201611001067

C) Owned by the authors, published by EDP Sciences, 2016

\title{
NUMERICAL ANALYSIS OF HEAT TRANSFER IN FIRE- PROTECTIVE COATINGS DEFORMABLE UPON HEATING
}

\author{
V.P. Rudzinsky ${ }^{1, a}$ and A.N. Garashchenko ${ }^{2}$ \\ ${ }^{1}$ National Research Tomsk Polytechnic University, 634050 Tomsk, Russia \\ ${ }^{2}$ Central Research Institute of Special Machinery, JSC, Khotkovo, Moscow Region, Russia
}

\begin{abstract}
Numerical studies of heat transfer in fire-protective coatings deformable (intumescent) upon heating have been conducted. The optimum combination of the computation-scheme parameters providing stability, convergence and satisfactory accuracy of solutions has been determined. An effect of basic characteristics of materials in real range of their change that made it possible to estimate the degree of influence of properties on the fireprotective efficiency of coatings and the level of warm-up (flame resistance) of structures to be protected with them has been studied. The possibility of using developed models and techniques to estimate and provide the required level of fire safety of polymer-based materials (in particular, elastomers and structures and products on their basis) is considered. The results of estimating the mass rate of evolving gaseous thermal-decomposition products that determine, in a considerable extent, the material combustibility have been presented. The numerical analysis results have demonstrated the potentiality of reducing the combustibility of such materials and increasing limits of their fire resistance at the expense of organizing the intumescence of a material upon heating by means of modification of their initial formulations as well as with the aid of an additional layer made of the intumescent coating compatible with an elastomer.
\end{abstract}

The numerical simulation of heat-mass-transfer processes in polymer-based fire-protective coatings (PFPC) which undergo heating up to temperatures of 1200-1300K typical for fire conditions and are deformed with high intensity upon heating has been performed. The studies have been carried out using a computer program developed by the numerical realization of an original mathematical model that the most fully described in $[1,2]$. The factors, which were not before considered, affecting the change dynamics of the forming swollen layer thickness: surface erosion as a result of thermochemical interaction between coke-foam carbon and gas medium, as well as time reduction of the thickness of the intumescent layer at the expense of its shrinkage (settlement) as the quantity of gaseous products of the thermal decomposition of a polymer base of materials passing by transit through the coke-foam layer is reduced, were taken into account.

\footnotetext{
${ }^{a}$ Corresponding author: a.n.gar@mail.ru
} 
The purpose of the numeral realization of an original model and the development of a computer program is the determination of basic laws for the processes under studied: dependencies of temperature on the interface "structure to be protected - fire-protective material" on characteristics of heat-conduction and radiation processes running in the coke layer of the material. The effect of physical-chemical and thermochemical processes in the material to be heated and on its surface on the intensity of heat transfer through the coke foam layer was analyzed. The nonstationarity of heat conduction processes under conditions at issue, as well as the nonlinearity of characteristics of materials under studied were taken into account.

An algorithm developed early for solving heat-conduction problems under intensive physicalchemical transformation conditions [3,4] was taken as a base in the numerical analysis. Numerical solving the problem was performed by a finite difference method using an algorithm providing the stability and convergence of the computational process in the presence of "disturbing" factors associated with features of processes to be simulated under high-temperature action (thermal destruction of a material, presence of movable boundaries of typical zones, presence of movable boundary between a coating and high-temperature gas medium and others).

Numerical studies, conducted in two directions, have been carried out. Firstly, the optimum combination of difference scheme parameters providing the stability and convergence of solutions under conditions of high-temperature action on PFPC was determined. Mesh widths of the difference grid on a coordinate and in time, their number, the change range as well as the iteration process unbinding value were determined. Secondly, the degree of influence of each-model parameters on the numeral simulation results was estimated and the parameters taking the most effect on the fire protection efficiency were disclosed. So, in particular, the coefficients which could not be determined in the course of direct experiments and which is not changed when changing over from one conditions to other ones (conservative to changing in practically important range of conditions of the hightemperature action on PFPC) were determined. These coefficients were accepted as the mathematical model parameters (radiant heat transfer in coke foam pores $\Theta$ and reduction of the coke foam thickness (burnout) $\psi$ ) [1, 2].

When solving the first problem, the optimum combination of grid parameters providing the undoubted convergence of the iteration process and computation time was determined. With this, the real ranges of characteristics of materials under investigation and regimes of high-temperature action on them were taken into account.

The results of numerical studies of heat transfer processes in the fire protection under the action of the gas flow whose temperature was changed under typical temperature condition, on it are given as an example. It is established that at the 45 -fold intumescence in calculations with the action time up to $60 \mathrm{~min}$, the mesh width in time should be not more than $0.001 \mathrm{~s}$, and in a coordinate - not more than $0.05 \mathrm{~mm}$. The necessary number of mesh widths in a coordinate in the material destruction and deformation (intumescence) zone (at least 200) has been ascertained. A mesh width range in time that is optimum for the used variant of computerized selection of a mesh width in the course of calculations has been determined. The possibility of considerable increase of mesh widths in time from initial $0.001 \mathrm{~s}$ to $0.5 \ldots 1 \mathrm{~s}$ in the subsequent period (intensity of processes taking place in PFPC is considerably reduced) has been established.

When solving the second problem, the effect of basic characteristics of materials in their real range was studied. The results extending the knowledge of heat transfer processes in materials under consideration have been obtained. The weak influence of values of the thermal effect of the thermal material destruction, specific heat capacity of gaseous thermal-destruction products as well as a kind of dependence of the PFPC decomposition degree on temperature has been ascertained. This result can be explained by relatively higher duration and relatively low intensity of the high-temperature action on the fire protection. At the same time, the numerical studies have clearly demonstrated a substantial role of the thickness of the intumescent layer (coke foam) and a dynamics of its change. The latter is determined, on the one hand, by the multiplicity and the PFPC intumescence temperature level and, on the other hand, by coefficient $\psi$ determining a dynamics of reducing its thickness [1, 2], which takes place owing to the surface erosion and settlement of the coke foam. Is also established the 
role of the coke-foam heat conduction that at the considerable temperature level is determined, in general, by the value of coefficient $\Theta$ in the expression for the coke foam heat conduction $[1,2]$. It has predetermined the selection of coefficients $\Theta$ and $\psi$ as parameters of the mathematical fireprotection model.

The influence of material properties on the heat transfer process was studied using, as an example, a model coating for which a range of changing these PFPC properties from the base variant was given. The warm up of steel structure with the reduced thickness of metal (cross section area-to-heat perimeter ratio) equal to $4 \mathrm{~mm}$ was simulated. The standard temperature fire regime was considered [1]. Some from results obtained are presented in Figures $1 \& 2$. It is ascertained that the multiplicity of the coating intumescence $\varepsilon_{v}$ (Figure 1a), parameter $\Theta$ (Figure 1b) and parameter $\psi$ (Figure 2a) that were entered in models $[1,2]$, exert the most influence on the efficiency of coatings.

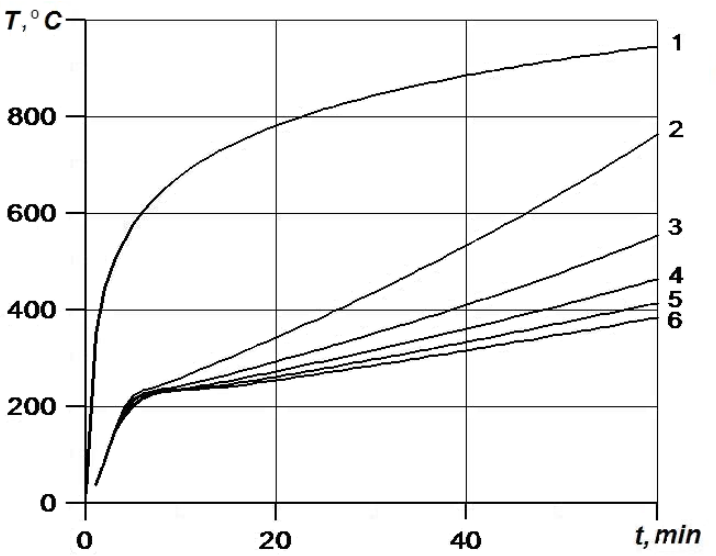

a)

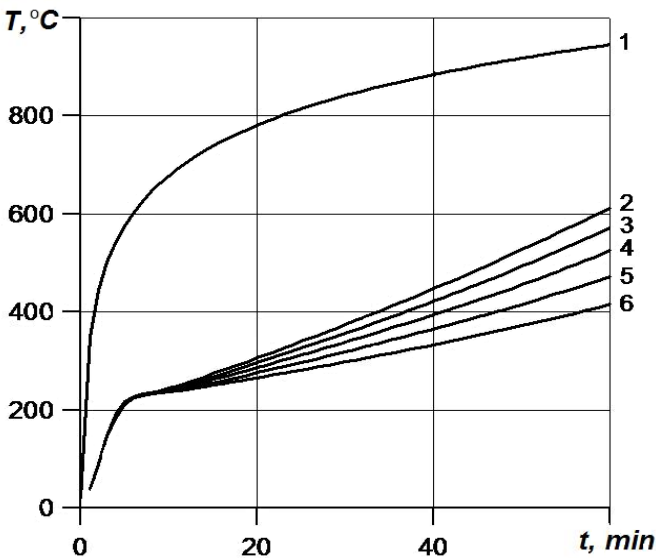

b)

Figure 1. Time dependences of temperature of gas medium (1) and steel structures with intumescence multiplicity (a) equal to 20 (2), 40 (3), 60 (4), 80 (5), 100 (6) and values of parameter $\Theta$ (b) equal to 20 (2), $2 \cdot 10^{-11}(3), 4 \cdot 10^{-11}(4), 7 \cdot 10^{-11}(5), 10 \cdot 10^{-11}(6) \mathrm{W} / \mathrm{mK}^{4}$.

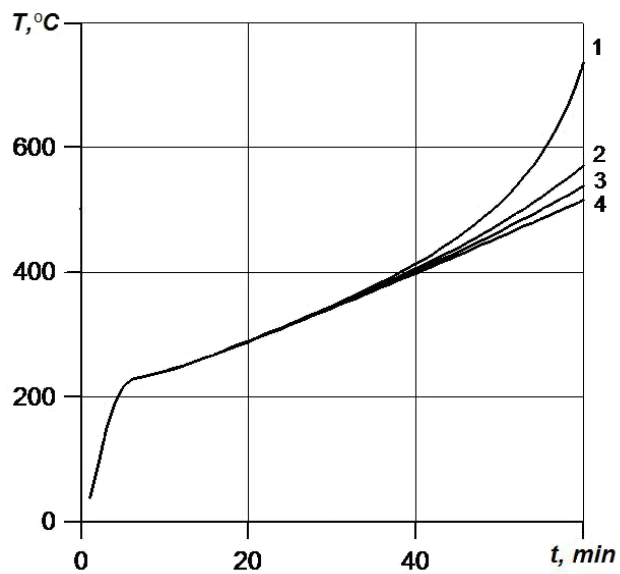

a)

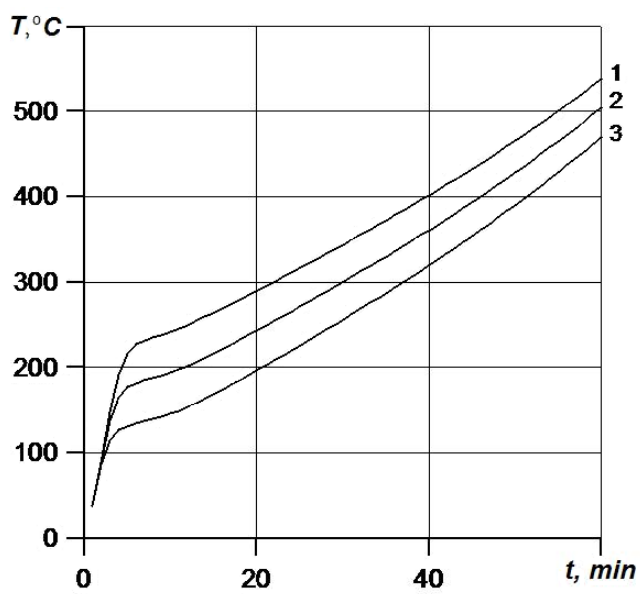

b)

Figure 2. Time dependences of temperature of steel structures at values of parameter $\psi$ (a) equal to 1.0 (1), 0.5 (2), 0.25 (3), 0 (4) and values of the intumescence onset temperature (b) equal to $220^{\circ} \mathrm{C}(1)-$ a basic variant, $170^{\circ} \mathrm{C}(2), 120^{\circ} \mathrm{C}(3)$. 
The results show a necessary of forming the coke foam with the considerable thickness, minimum surface removal (shrinkage) and stable fine-porous structure. Also, the substantial role of intumescence temperatures (Figure $2 b$ ) is shown. The intumescence onset temperature substantially influence the warm-up level of structures to be protected that is evidence of the importance of its reduction.

Another possible application of developed models and techniques [1,2] covering problems of providing a high level of fire safety of polymer-based materials (in particular, elastomers and structures and products thereof) is considered. Reducing the combustibility in such materials and increasing limits of their fire resistance at the expense of organizing the material intumescence upon heating, that is reachable in the case of the corresponding modification of their original formulations, are prospective. Mass rates of evolving combustible gaseous thermal-destruction products which determine, to a great extent, the combustibility of materials have been estimated.

The numerical studies have been also conducted in the presence of the additional surface layer of the intumescent coating (SGK-2 composition with 25-fold intumescence) compatible with an elastomer. The results of simulating the destruction depth (advances of isotherm $300^{\circ} \mathrm{C}$ ) and the rate of gas evolving from a modified elastomer in the absence or in the presence of the additional PFPC on the surface are presented in Figures $3 \& 4$. Figure 3 shows destruction depths and gas evolving rates for different multiplicity of surface-layer intumescence in material in the absence of a layer of additional fire protection. Similar results were also obtained in the presence of the different-thickness PFPC under study on the surface (Figure 4).

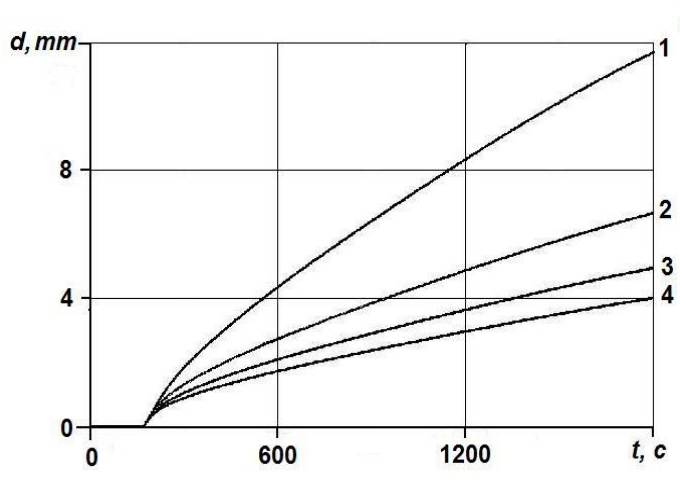

a)

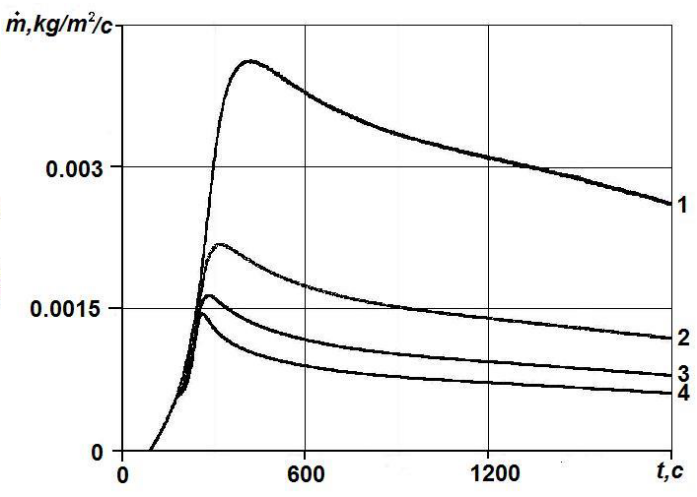

b)

Figure 3. Time histories of destruction depth (a) and mass rate of evolving combustible gaseous thermaldestruction products (b) for an elastomer with intumescence multiplicity of 0 (1), 2 (2), 4 (3), 6 (4).

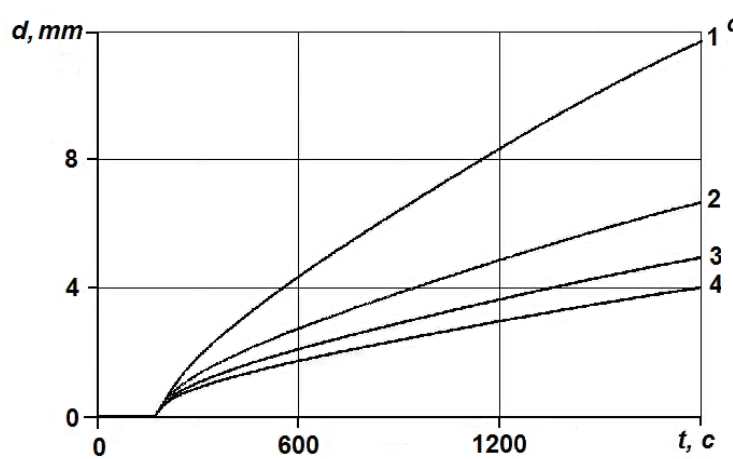

a)

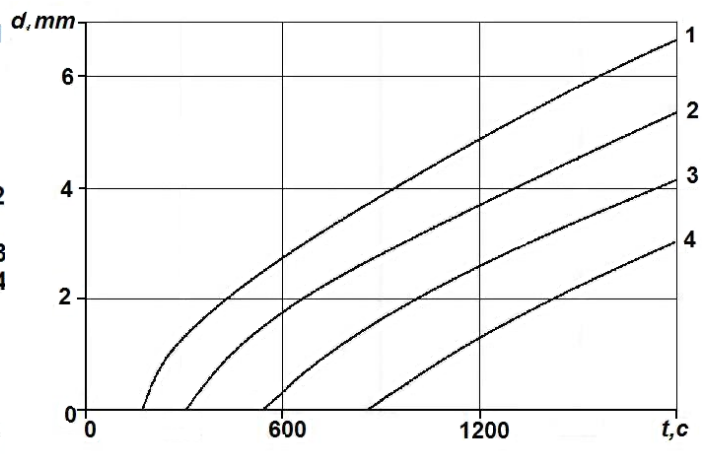

b) 


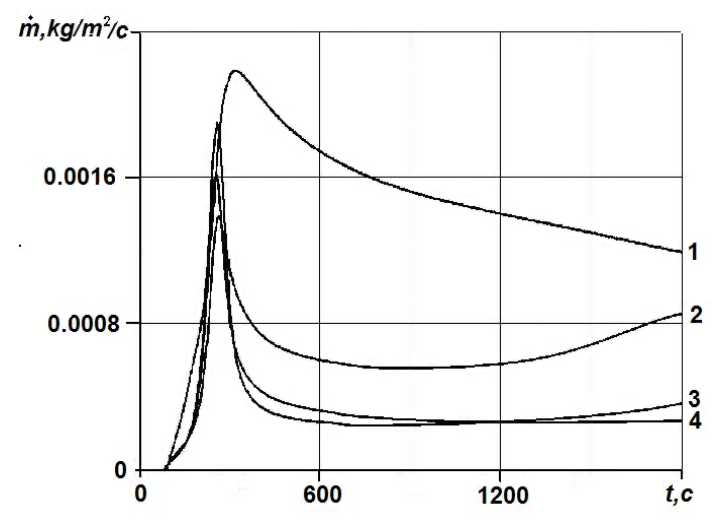

c)

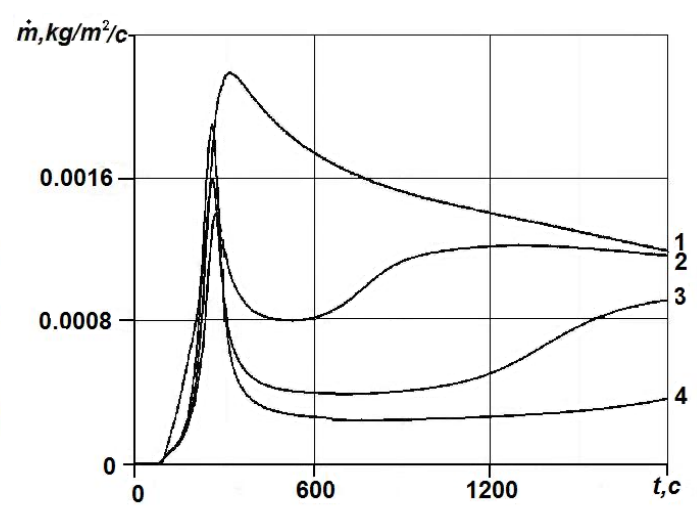

d)

Figure 4. Time histories of destruction depth $(a, b)$ and mass rate of evolving combustible gaseous thermaldestruction products $(\mathrm{c}, \mathrm{d})$ for an elastomer with intumescence multiplicity equal to 2 in the presence of intumescent coating $0.5 \mathrm{~mm}(\mathrm{a}, \mathrm{b})$ and $1 \mathrm{~mm}(\mathrm{c}, \mathrm{d})$ thick.

The numerical studies have been shown that it is possible three-fold reduction of the gas evolving rate of a material at the expense of the elastomer intumescence, and the reduction almost by a factor of 10 in the presence of an additional coating. As is obvious from Figures $3 \& 4$, a level of the elastomer destruction depth, in a corresponding way, is also considerably reduced that is evidence of increasing the fire-resistance limit of a structure. It is also shown that in the presence of PFPC under specific conditions, the material will not destructed during the long action time. The obtained results (Figures $3 \& 4$ ) illustrate new possibilities of increasing fire safety indices of polymer-based materials and fireresistance limits of corresponding structures and products.

\section{References}

1. V.L. Strakhov, A.N. Garashchenko, G.V. Kuznetsov, V.P. Rudzinsky, Mathematical Modeling, 12 (5), 107 (2000)

2. V.L. Strakhov, A.N. Garashchenko, G.V. Kuznetsov, V.P. Rudzinsky, Combustion, Explosion, and Shock Waves, 37 (2), 212 (2001)

3. G.V. Kuznetsov, V.P. Rudzinsky, Combustion, Explosion, and Shock Waves, 34 (3), 84 (1998)

4. G.V. Kuznetsov, V.P. Rudzinsky, J. Appl. Mech. Tech. Phys., 40 (3), 143 (1999) 